\title{
Management of Class II Division I Malocclusion with Carriere Motion Appliance: A Case Report
}

\author{
Pavithranand Ammayappan ${ }^{1}$, Arulmani Periasamy ${ }^{2}$
}

\begin{abstract}
Most of the patients reporting for the correction of proclined teeth have an underlying skeletal Class II base relation; management of these cases during the growth phase early in the treatment when patient compliance is at their best is advantageous. Although various functional appliances are available for use, the Carriere Motion Appliance (CMA) is simple, not bulky, and easy for the patient to adapt, as there is no bulky acrylic that would hinder the speech or during eating. This case report presents a 14-year-old patient treated with the CMA.

Keywords: Carriere Motion Appliance, Class II malocclusion, Patient cooperation.

Journal of Scientific Dentistry (2020): 10.5005/jp-journals-10083-0930
\end{abstract}

The most common chief complaint of patients reporting to the clinics for orthodontic treatment, other than crowded teeth, is proclined upper anteriors. ${ }^{1}$ This condition is usually associated with an underlying Class II skeletal base, which can be corrected nonsurgically during the growth phase of an individual, with various functional appliances like the Twin Block appliance, Herbst, Activator, Headgear, etc. The problems with most of these are that the patients take a while getting accustomed to these appliances due to the bulky acrylic and the interference to their speech and mastication.

Carriere Motion Appliance (CMA) was introduced in the early 2004 as a distalizer, for the correction of the Class II malocclusion. ${ }^{2}$ The appliance consists of a metal rod like device with mesh pads at either end of it for bonding the appliance onto the molar and the cuspid (or first premolar or first deciduous molar) an attachment on the lower molar for engagement of elastics to the cuspid. The lower arch anchorage is reinforced by means of a lingual arch or Essix retainer.

This case report presents a growing male patient, treated with the CMA with minimal discomfort and good patient compliance.

\section{Diagnosis and Treatment Plan}

A 14-year-old male reported with a chief complaint of prominent upper front teeth. He presented with a convex profile and potentially competent lips; the profile was exacerbated due to the deep mentolabial sulcus, lip trap, and the strong chin. The patient presented with a Class II skeletal pattern with the molars and cuspids in a Class II relation on both sides and a deep curve of Spee, the
${ }^{1}$ Professor and Head, Department of Orthodontics and Dentofacial Orthopaedics, Indira Gandhi Institute of Dental Sciences, Sri Balaji Vidyapeeth, Puducherry, India

${ }^{2}$ Private Practitioner, SV Dental Clinic, Salem, Tamil Nadu, India

Corresponding Author: Pavithranand Ammayappan, Professor and Head, Department of Orthodontics and Dentofacial Orthopaedics, Indira Gandhi Institute of Dental Sciences, Sri Balaji Vidyapeeth Puducherry, India, Phone: +91 9443183590, e-mail: docanandpavi@ gmail.com

How to cite this article: Ammayappan P, Periasamy A. Management of Class II Division I Malocclusion with Carriere Motion Appliance: A Case Report. J Sci Dent 2020;10(2):43-45.

Source of support: Nil

Conflict of interest: None

maxillary arch was constricted and crowding was evident in the mandibular arch, and the upper incisors were protrusive with an overjet of $11 \mathrm{~mm}$ and a deep bite of $6 \mathrm{~mm}$ (Fig. 1).

Taking into consideration the growth potential of the patient, it was decided to start the treatment in two phases with a functional appliance therapy during phase 1 to achieve Class I relation followed by the phase 2 treatment involving complete fixed orthodontic appliance for correction of the proclination. The patient and his parents were provided two options for the functional phase: either with Twin Block appliance or the newer CMA. The parents chose the CMA over the Twin Block as they were not sure about the compliance of their ward with the Twin Block.
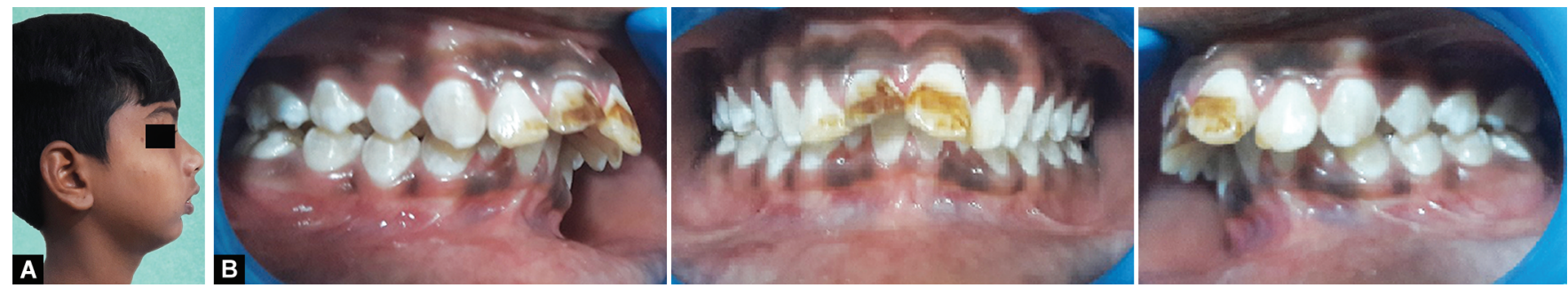

Figs 1A and B: (A) Pretreatment extraoral; (B) Pretreatment intraoral

(0) The Author(s). 2020 Open Access This article is distributed under the terms of the Creative Commons Attribution 4.0 International License (https://creativecommons. org/licenses/by-nc/4.0/), which permits unrestricted use, distribution, and non-commercial reproduction in any medium, provided you give appropriate credit to the original author(s) and the source, provide a link to the Creative Commons license, and indicate if changes were made. The Creative Commons Public Domain Dedication waiver (http://creativecommons.org/publicdomain/zero/1.0/) applies to the data made available in this article, unless otherwise stated. 

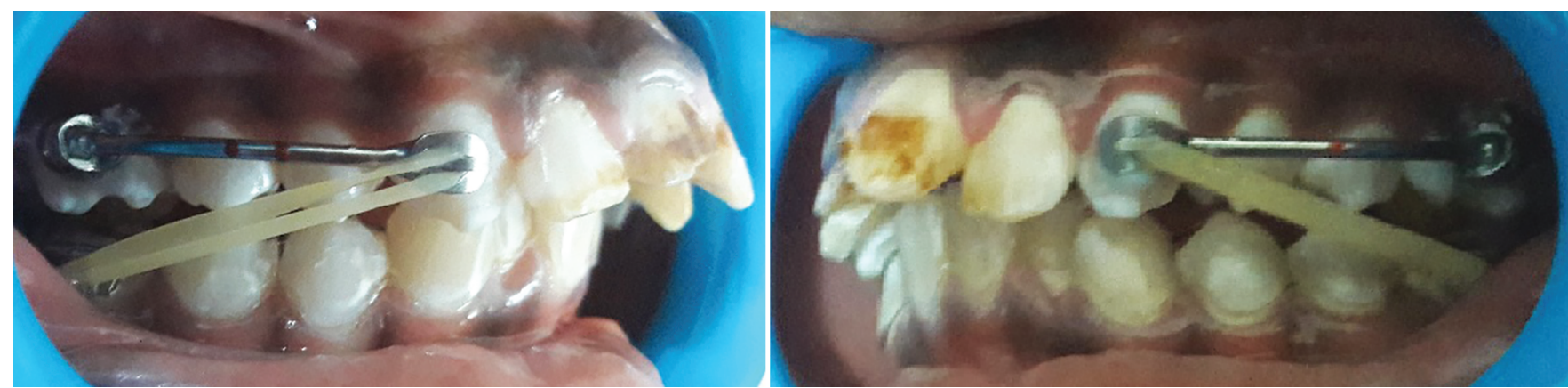

Fig. 2: With appliance
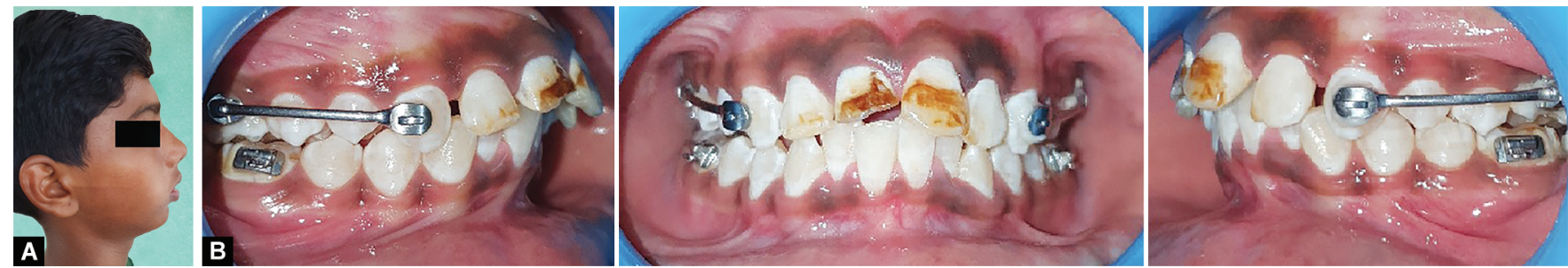

Figs $3 \mathrm{~A}$ and B: (A) Posttreatment extraoral; (B) Posttreatment intraoral

\section{Treatment Progress}

The treatment was initiated by bonding of the CMA in the upper arch - between the first molar and the cuspid on both sides (Fig. 2). Buccal tubes were bonded on the lower first molars for engaging elastics and for anchorage purpose; an Essix retainer for the lower arch was delivered. The patient was instructed to wear the elastics full-time. Initially treatment was started with the 6 oz, 1/4" Class II elastics for the first month following which $8 \mathrm{oz}, 3 / 16$ " Class II elastics were prescribed. After a 2-week recall, it was found out that the patient was noncompliant with the elastic wear and after further counseling the patient was regular with the elastic protocol. Class I molar and canine (on left side) was achieved in a period of 5 months (Fig. 3). The overjet also reduced to $4 \mathrm{~mm}$. The CMA was debonded in the subsequent month. The patient's father was happy with the result and refused to continue with the phase 2 treatment.

\section{Treatment Results}

After 6 months of treatment with the CMA, a bilateral Class I molar and cuspid relation was achieved. The overjet and overbite were also significantly decreased. Spaces opened mesial to the cuspids on both sides (as was expected) due to its distalization because of the use of Class II elastics. There was a decrease in the convexity of the profile and only a slight improvement in the competency of the lips as the patient failed to initiate his phase 2 treatment.

\section{Discussion}

Nonsurgical management of Class II malocclusion involves either a two-phase treatment where there is the use of functional appliance for correcting the Class II skeletal base as a removable appliance during Phase 1 treatment followed by fixed appliance for dental correction $^{3,4}$ or the use of a fixed functional appliance along with the existing fixed orthodontic appliance. ${ }^{4}$ Although the fixed functional appliance does not rely on patient compliance, it usually restricts mandibular movement or can cause injuries when broken. The removable functional appliance is usually well tolerated by the patients but patient cooperation should be at the maximum level for achieving proper results.

It is claimed by the developer that with the use of CMA, the mandible is repositioned anteriorly into a more favorable position and the Class II correction is also achieved by the distal movement of the entire posterior segment from the molar to the cuspid and a distal tipping of the molar. Various studies have reported that the Class II correction with CMA is only achieved by the dentoalveolar correction with little or no significant effect can be seen in the mandible. ${ }^{5,6}$ These results are also in accordance with other studies that evaluated the effects of various other functional appliances. ${ }^{7,8}$

The CMA is chiefly a fixed appliance and the only removable components are the elastics and the Essix retainer. In our practice, it is well tolerated by the patients and is preferred over other functional appliances like the Twin Block, and good compliance can be expected with the use of elastics as they are needed to be worn only in initial period of the treatment when cooperation is at maximum level. The Class II correction is achieved within 6 months of the treatment following which routine orthodontic dental correction can be achieved in a relatively short duration of time.

\section{References}

1. Maheshwaran T, Ramesh V, Krishnan A, Joseph J. Common chief complaints of patients seeking treatment in the government dental institution of Puducherry, India. J Indian Acad Dent Spec Res 2015;2(2):55-58.

2. Carriere L. A new class II distalizer. J Clin Orthod 2004;38(4):224-231.

3. Nayak KU, Goyal V, Malviya N. Two-phase treatment of class II malocclusion in young growing patient. Contemp Clin Dent 2011;2(4):378-380.

4. Profitt WR, Fields HW, Sarver DM. Contemporary Orthodontics. 4th ed., Mosby Elsevier; 2007. pp. 510-534.

5. Kim-Berman H, McNamara Jr JA, Lints JP, McMullen C, Franchi L. Treatment effects of the carriere motion 3D appliance for the correction of class II malocclusion in adolescents. And Orthod 2019;89(6):839-846. DOI: 10.2319/121418-872.1.

6. Yin K, Han E, Guo J, Yasumura T, Grauer D, Sameshima G. Evaluating the treatment effectiveness and efficiency of carriere distalizer: a 
cephalometric and study model comparison of class II appliances. Prog Orthod. 2019;20(1):24. DOI: 10.1186/s40510-019-0280-2.

7. Collett AR. Current concepts on functional appliances and mandibular growth stimulation. Aust Dent J 2000;45(3):173-178. DOI: 10.1111/ j.1834-7819.2000.tb00553.x.
8. Zymperdikas VF, Koretsi V, Papageogiou SN, Papadopoulos $M A$. Treatment effects of fixed functional appliances in patients with class II malocclusion: a systematic review and metaanalysis. Eur J Orthod 2016;38(2):113-126. DOI: 10.1093/ejo/ cjv034. 